\title{
AVALIAÇÃO NUTRICIONAL PRÉ E PÓS-OPERATÓRIA EM PACIENTES COM MEGAESÔFAGO NÃO-AVANÇADO
}

\author{
Maria Aparecida Coelho de Arruda HENRY1, Vânia Cristina LAMONICA-GARCIA² e \\ Mauro Masson LERCO ${ }^{1}$
}

\begin{abstract}
RESUMO - O megaesôfago, afecção caracterizada por aperistalse do corpo esofágico e relaxamento deficiente do esfíncter inferior do esôfago, apresenta a disfagia como o sintoma mais frequente. O objetivo deste estudo foi avaliar o estado nutricional de pacientes com megaesôfago não-avançado nos períodos pré e pós-operatórios de cardiomiotomia videolaparoscópica. Dez pacientes foram avaliados em cinco momentos (pré-operatório e aos 1, 3,6 12 meses após a cirurgia). Os parâmetros antropométricos, hematimétricos e bioquímicos foram estudados nos cinco momentos. Conclusões: 1) a maioria dos pacientes com megaesôfago não-avançado é eutrófica; 2) o tratamento cirúrgico acarreta melhora do estado nutricional e aumento dos valores do HDL colesterol.
\end{abstract}

DESCRITORES - Acalásia esofágica, cirurgia. Avaliação nutricional. Cuidados pré-operatórios. Cuidados pós-operatórios.

\section{INTRODUÇÃO}

O megaesôfago, doença caracterizada por distúrbio motor do corpo esofágico e do esfíncter inferior, constitui sério problema de saúde publica em nosso país ${ }^{(1,2)}$.

A disfagia é o sintoma predominante, a qual compromete a qualidade de vida e o estado nutricional, em decorrência da inadequada ingestão alimentar ${ }^{(3,5)}$.

Este estudo teve por objetivos avaliar o estado nutricional de pacientes com megaesôfago nãoavançado e investigar o efeito do tratamento cirúrgico para tal afecção.

\section{MÉTODOS}

Foram estudados 10 pacientes ( 6 homens e 4 mulheres), com idades entre 34 e 76 anos (média: $53 \pm 12,9$ anos). $\mathrm{O}$ diagnóstico de megaesôfago foi baseado nos achados clínicos, sorológicos, radiológicos, endoscópicos e manométricos. Cinco pacientes apresentavam megaesôfago grau III, quatro com megaesôfago grau I e um doente grau II.

Os pacientes foram operados segundo cardiomiotomia videolaparoscópica associada à válvula antirefluxo gastroesofágico ${ }^{(1,4)}$.

Os pacientes foram submetidos a avaliação nutricional envolvendo aspectos antropométricos, hematimétricos e bioquímicos, realizada antes da cirurgia (momento 1 - M1 -) e aos 1, 3, 6 e 12 meses após o procedimento (momentos 2, 3, 4 e 5-M2, M3, M4, e M5-).

\section{RESULTADOS}

1. Avaliação nutricional pré-operatória a. As médias e desvios-padrão referentes aos parâmetros peso corporal, índice de massa corpórea (IMC), percentagem de gordura corporal $(\% \mathrm{GC})$ e circunferência muscular do braço estão na Tabela 1 (M1).

b. Com relação aos parâmetros hematimétricos observados no período pré-operatório, os valores médios foram: contagem total de linfócitos (CTL) $2170 \pm 682$ células $/ \mathrm{mm}^{3}$, hematócrito (Hct) $44,2 \pm$ $3 \%$ e hemoglobina $(\mathrm{Hb}) 14,8 \pm 1,0 \mathrm{~g} / \mathrm{dL}$.

c. Parâmetros bioquímicos: valores médios

\begin{tabular}{|c|c|}
\hline Albumina: $4,3 \pm 0,4 \mathrm{mg} / \mathrm{DL}$ & colesterol total: $199 \pm 38 \mathrm{mg} / \mathrm{DL}$ \\
\hline LDL colesterol: $135 \pm 35 \mathrm{mg} / \mathrm{DL}$ & HDL colesterol: $48 \pm 11 \mathrm{mg} / \mathrm{DL}$ \\
\hline
\end{tabular}

2. Avaliação nutricional pós-operatória

a. O peso corpóreo apresentou redução dos seus valores no momento 2 , porém a partir dos 3 meses, os pacientes recuperaram o peso, havendo elevação do mesmo $(P<0,05)$. A \% GC mostrou-se reduzida no $1^{\circ}$ mês de pós-operatório, com recuperação a partir do $3^{\circ}$ mês.

IMC: Discreta redução do mesmo em M2, com recuperação nos meses subsequentes (Tabela 1).

CMB: Não mostrou alteração no pós-operatório. b. Parâmetros hematimétricos - com relação a CTL, Hct e Hb, não foram observadas alterações

\footnotetext{
Trabalho realizado no Departamento de Cirurgia e Ortopedia da Faculdade de Medicina de Botucatu, Universidade Estadual Paulista - UNESP, Botucatu, SP.

Departamento de Cirurgia e Ortopedia da Faculdade de Medicina de Botucatu, Universidade Estadual Paulista - UNESP; ${ }^{2}$ Curso de Pós-Graduação Bases Gerais da Cirurgia da Faculdade de Medicina de Botucatu, Universidade Estadual Paulista - UNESP.

Correspondência: Profa. Maria Aparecida Coelho de Arruda Henry - Rua Miguel Ciofi, 200 - 18607-790 - Botucatu, SP. Email: rhenry @ibb.unesp.br
} 
TABELA 1. Avaliação do estado nutricional de acordo com a antropometria. Comparação entre as médias e desvios-padrão nos cinco momentos de estudo

\begin{tabular}{|c|c|c|c|c|}
\hline \multicolumn{5}{|c|}{ Parâmetros } \\
\hline Momentos & Peso & IMC & $\% \mathrm{GC}$ & CMB \\
\hline M 1 & $61,3 \pm 10,2 \mathrm{ab}$ & $23,4 \pm 4,1 b$ & $26,9 \pm 10,2 \mathrm{ab}$ & $25,3 \pm 2,4$ \\
\hline M 2 & $59,9 \pm 9,2 \mathrm{~b}$ & $22,8 \pm 3,5 b$ & $26,4 \pm 9,5 b$ & $24,8 \pm 2,5$ \\
\hline M 3 & $61,7 \pm 7,6 \mathrm{ab}$ & $23,5 \pm 2,9 a$ & $27,4 \pm 9,8 \mathrm{ab}$ & $25,0 \pm 1,9$ \\
\hline M 4 & $62,6 \pm 7,2 \mathrm{a}$ & $23,9 \pm 3,7 \mathrm{a}$ & $28,8 \pm 9,4 \mathrm{ab}$ & $25,2 \pm 1,6$ \\
\hline M 5 & $63,4 \pm 7,2 \mathrm{a}$ & $24,3 \pm 3,4 \mathrm{a}$ & $28,8 \pm 11,4 \mathrm{a}$ & $25,0 \pm 1,7$ \\
\hline Estatística & $P<0,05$ & $P<0,05$ & $P<0,05$ & $P>0,05$ \\
\hline
\end{tabular}

IMC $=$ índice de massa corporal

$\% \mathrm{GC}=$ percentagem de gordura corpor

$\mathrm{CMB}=$ - pircunfêtecia muscular do braço

dos mesmos no período pós-operatório, em qualquer dos momentos estudados.

c. Parâmetros bioquímicos - As dosagens de albumina, colesterol total e LDL colesterol realizadas no pósoperatório não mostraram alteração quando comparadas com os valores pré-operatórios $(P>0,05)$. O HDL colesterol apresentou elevação de seus valores no pós-operatório $(P<0,05)$.

\section{DISCUSSÃO}

A metodologia usada na presente pesquisa permitiu avaliar de maneira satisfatória o estado nutricional dos pacientes com megaesôfago não-avançado.

A análise dos parâmetros antropométricos mostrou que no pré-operatório, 9 dos 10 pacientes apresentavam-se eutróficos, sendo que o único com depleção dos tecidos adiposo e muscular era o mais idoso do grupo, portanto já apresentando as alterações próprias do envelhecimento. Este resultado deve ser decorrente da precocidade com que o diagnóstico e tratamento foram realizados nestes pacientes, pois naqueles com megaesôfago avançado estudados por PENHAVEL et al. ${ }^{(5)}$ foi observado acentuado grau de desnutrição.
Com relação aos parâmetros hematimétricos e bioquímicos, apenas um doente mostrou discreta depleção de linfócitos e albumina, que não deve ser decorrente da doença de base.

No pós-operatório precoce (M2) os pacientes apresentaram redução do peso corpóreo, IMC e \% GC, sem alteração significativa da CMB. Este resultado pode ser interpretado como resposta à fase catabólica do estresse cirúrgico, ao lado de ingestão insuficiente de nutrientes na fase inicial do período pós-operatório.

Na avaliação pós-operatória tardia houve sensível recuperação do peso corpóreo, IMC e \% GC. Este resultado mostra que os pacientes se beneficiaram com o tratamento cirúrgico, com possível melhora da qualidade de vida, conforme referido por CECCONELLO et al. (2).

Com relação aos parâmetros hematimétricos e bioquímicos, não foi observada alteração nas fases precoce e tardia do período pós-operatório, com exceção de HDL colesterol, que mostrou elevação de seus valores $(P<0,05)$.

Os resultados do presente estudo permitem concluir que:

1. A maioria dos pacientes com megaesôfago não-avançado apresenta-se eutrófica;

2. O tratamento cirúrgico ocasiona melhora do estado nutricional e elevação dos valores do HDL colesterol.

Henry MACA, Lamonica-Garcia VC, Lerco MM. Preoperative and postoperative nutrional evaluation in patients with non-advanced megaesophagus. Arq Gastroenterol. 2009;46(4):341-2.

ABSTRACT - Megaesophagus, an affection characterized by the aperistalsis of the esophageal body and deficient relaxation of the lower esophageal sphincter presents dysphagia as the most frequent complaint. The goal of this study was to evaluate the nutritional status of patients with nonadvanced megaesophagus in pre and postoperative periods of laparoscopic cardiomyotomy. Ten patients were studied in five moments (pre operative and at 1, 3, 6 and 12 months after surgery). The anthropometric, hematimetric and biochemical parameters were studied in five moments. Conclusions: 1) most patients with non-advanced megaesophagus were eutrophic; 2) surgical treatment led to improvement in nutritional status and increase of HDL cholesterol fraction.

HEADINGS - Esophageal achalasia, surgery. Nutritional assessment. Preoperative care. Postoperative care.

\section{REFERÊNCIAS}

1. Andreollo NA, Lopes LR, Coelho-Neto JL, Camargo MA. Tratamento cirúrgico do megaesôfago não avançado: laparotomia ou videolaparoscopia? GED Gastroenterol Endosc Dig. 2007;26:194-9.

2. Cecconelo I, Santo MA, Szachnowicz S, Takeda FR, Namur GN, Gama-Rodrigues J. Avaliação da qualidade de vida em pacientes com megaesôfago chagásico submetidos a tratamento cirúrgico individualizado. ABCD Arq Bras Cir Dig. 2004; 17:127-30.

3. Meneghelli UG, Dantas RO. Avaliação clínica da motilidade esofagiana. In:
Mincis M, editor. Gastroenterologia e hepatologia - diagnóstico e tratamento. São Paulo: Lemos; 1997. p.219-26.

4. Mineo TC, Ambrogi V. Long-term results and quality of life after surgery for oesophageal achalasia: one surgeon's experience. Eur J Cardiothoracic Surg. 2004;25:1089-96.

5. Penhavel FAZ, Waitzberg DL, Trevenzol HP, Alves L, Zilberstein B, GamaRodrigues J. Pre-and postoperative nutritional evaluation in patients with chagasic megaesophagus. Nutr Hosp. 2004;19:89-94

Recebido em 23/4/2008 Aprovado em 28/7/2008 